\title{
DESSORÇÃO, EXTRAÇÃO E FRACIONAMENTO DE MANGANÊS EM LATOSSOLOS ${ }^{(1)}$
}

\author{
C. W. A. NASCIMENTO(2), R. L. F. FONTES ${ }^{(3)} \&$ J . C. L. NEVES ${ }^{(3)}$
}

\begin{abstract}
RESUMO
A pesquisa sobre a avaliação da disponibilidade de manganês tem apresentado resultados discordantes, em grande parte em decorrência dos teores considerados disponíveis pelos diversos extratores. $\mathrm{O}$ conhecimento das formas químicas em que se encontra o elemento e suas relações com os teores disponíveis são importantes para a previsão do seu comportamento no sistema solo-planta. Nesse sentido, este trabalho teve como objetivo estudar a influência da calagem e de doses de manganês sobre a dessorção, extração e fracionamento desse elemento em amostras de seis Latossolos. Essas amostras, submetidas ou não à calagem, receberam o manganês nas doses de 0,16 e $32 \mathrm{mg} \mathrm{dm}^{-3}$ e permaneceram incubadas por 30 dias. Terminada a incubação, o Mn foi determinado utilizandose os extratores Mehlich-1, Mehlich-3, DTPA e EDTA. As amostras foram submetidas a um fracionamento que separou o Mn na fração trocável (Tr), matéria orgânica (MO), óxido de manganês (OxMn), óxido de ferro amorfo (OxFeA), óxido de ferro cristalino $(\mathrm{OxFeC})$ e residual $(\mathrm{R})$, além dos teores totais. A dessorção foi avaliada mediante extrações sucessivas com resina de troca catiônica. Concluiuse que o elemento foi retido, principalmente, na fração trocável e matéria orgânica. De modo geral, a calagem provocou redução nos teores trocáveis e aumento nas frações óxidos de ferro amorfo e de manganês. Na ausência da calagem, a ordem de dessorção apresentada foi $\mathrm{Tr} \gg \mathrm{MO}>0 \times \mathrm{XFeA}$, com o Mehlich-3 sendo o melhor extrator para o Mn lábil. A calagem provocou acentuado decréscimo nos teores de Mn dessorvidos. 0 Mehlich-1 mostrou-se inadequado para a determi nação dos teor es disponíveis de Mn em solos corrigidos, condição na qual o DTPA foi o extrator mais indicado.
\end{abstract}

Termos de indexação: calagem, análise de solo, mi cronutriente.

(1) Recebido para publicação em setembro de 2001 e aprovado em abril de 2002.

(2) Professor do Departamento de Agronomia, Universidade Federal Rural de Pernambuco - UFRPe. Rua Dom Manuel de Medeiros s/n, Dois I rmãos, CEP 52171-900 Recife (PE). E-mail: cwan@bol.com.br

(3) Professor do Departamento de Solos, Universidade Federal de Viçosa - UFV. Av. PH Rolfs s/n, CEP $36571-000$ Viçosa (MG). E-mail: renildes@solos.ufv.br 


\title{
SUMMARY: DESORPTION, EXTRACTION AND FRACTIONING OF MANGANESE IN OXISOLS
}

\begin{abstract}
Conflicting results have been achieved by the evaluation of manganese availability in soil, largely a consequence of varying values denoted as avail able by different extractants. Knowl edgeon theelement's chemical forms in thesoil and on relationships with theavailable concentrations are important to predict its behavior in thesoil-plant system. In this study, six Oxisol soil samples were analyzed to evaluate the influence of liming and different manganese doses on desorption, extraction and fractioning. These soil samples, with and without li me application, were treated with manganese doses $\left(0.0,16.0\right.$ and $32.0 \mathrm{mg} \mathrm{dm}^{-3}$ ) and then incubated for 30 days. Hereupon, $\mathrm{Mn}$ was extracted with Mehlich-1, Mehlich-3, DTPA and EDTA. and determi ned by atomic adsorption spectrophotometry. Additionally, thesamples werefractionated for determination of manganesein thefractions: exchangeable $(E x)$, organic matter $(\mathrm{OM})$, manganese oxides $(\mathrm{MnOx})$, amorphous iron oxides ( $\mathrm{AFeOx}$ ), crystallineiron oxides ( $\mathrm{CFeOx}$ ), and residual (Re), as well as the total concentrations of the micronutrient. Micronutrient desorption was evaluated by successiveextractions with cation exchange resin. Results showed that $\mathrm{Mn}$ was mainly retained in the exchangeable and organic matter fractions. In general, liming reduced exchangeable contents and increased the oxidefractions of amorphous iron and manganese. Without lime, the desorption order for $\mathrm{Mn}$ was $\mathrm{Ex}>\mathrm{OM}>\mathrm{AFeOx}$, where Mehlich-3 proved to be the best extractor for the determination of labile $\mathrm{Mn}$ concentrations. Liming caused a considerable decrease in $\mathrm{Mn}$ desorption. Under the same condition, Mehlich-1 proved to be inefficient to determine available Mn contents, while DTPA was the best extractant.
\end{abstract}

Index terms: liming, soil analysis, mi cronutrient.

\section{NTRODUÇÃO}

A pesquisa sobre a avaliação da disponibilidade de manganês em solos tem, freqüentemente, apresentado resultados discordantes, em grande parte, em decorrência das diferenças nos teores considerados "disponíveis" pel os diversos extratores esuas relações com características dos sol os e formas químicas em que se encontra o el emento.

Os processos químicos mais importantes que influenciam o comportamento do Mn no solo e sua disponibilidade para as plantas são os que se relacionam com sua adsorção no solo, aceitando-se, geral mente que as concentrações na solução do solo e, conseqüentemente, a disponibilidade para as plantas são controladas por reações de sorçãodessor ção (Harter, 1991; Hogg et al., 1993). E mbora numerosos estudos tenham examinado a adsorção de micronutrientes em solos, especial mente de Cu e deZn (Shuman, 1975; Msaky \& Calvet, 1990; Bibak, 1997; Yuan \& Lavkulich, 1997; Mesquita, 1998), pouquíssimos têm-se dedicado ao processo reverso: dessorção desses elementos, sendo inexistentes na literatura consultada estudos sobre dessorção de manganês. Como observado por Swift \& McLaren (1991), tal situação é paradoxal, uma vez que a dessorção constitui o processo que controla a concentração e a taxa de fornecimento dos micronutrientes para a solução do solo.
Extrações químicas simples têm sido extensivamente utilizadas para medir os teores de $\mathrm{Mn}$ em solos. No entanto, como a quantidade do micronutriente extraída varia com o extrator, essas extrações têm apresentado graus variados de sucesso para diagnose de deficiência ou de toxidez desse elemento para as plantas. Segundo Zhu \& Alva (1993), a identificação das formas nas quais o micronutriente é extraído pel os diferentes métodos de extração pode ajudar a entender a eficiência de cada método.

Selecionar uma solução química que possa remover teores imediata epotencialmentedisponíveis de um nutriente requer entendimento das várias formas do elemento nos solos (Sims \& J ohnson, 1991). N esse sentido, estudos que utilizam técnicas de fracionamento, as quais indicam as formas de micronutrientes mais disponíveis às plantas ou que melhor se correlacionam com a disponibilidade indicada por métodos de extração, além de características dos solos e de manejo que influem nessa disponibilidade, exercem papel primordial na previsão do comportamento dos micronutrientes no sistema sol o-planta.

O pH do solo, na maioria dos casos, é o mais importante fator de controle da disponibilidade de $\mathrm{Mn}$ para as plantas, visto que esse elemento tem sua disponibilidade diminuída pela el evação do pH do sol o. O efeito da calagem sobre a disponibilidade 
se deve, principal mente, ao incremento dos sítios de adsorção no solo, graças à existência de cargas dependentes de $\mathrm{pH}$, como observado por Alloway (1990), e, por conseguinte, à densidade de cargas negativas presentes na superfície dos colóides. Portanto, para a definição da disponibilidade, é essencial conhecer como o pH influencia a distribuição de $\mathrm{Mn}$ entre as frações de solo.

Este trabal ho objetivou estudar a influência da calagem e de doses de manganês sobre a dessor ção, extração química e fracionamento desse el emento em amostras de Latosssolos.

\section{MATERIAL E MÉTODOS}

Amostras de seis solos (0-20 m) foram coletadas em muniápios doestado deMinas Gerais, sel ecionados de acordo com os teores diferentes de argila e de carbono orgânico. Esses solos foram classificados como Latossolo Vermelho - LV1 (Sete Lagoas), Latossol oVermel ho- LV2 (SãoSebastião do Paraíso), Latossol oVermel ho- LV3 (São Sebastião do Paraíso), LatossoloVermelho-Amarelo- LVA1 (J oão Pinheiro),
Latossolo Vermel ho-Amarelo - LVA2 (Viçosa) e Latossolo Vermel ho-Amarelo húmico - LVA3 (Viçosa) (Quadro 1).

As amostras dos solos foram homogeneizadas e passadas em peneira de $2,0 \mathrm{~mm}$ de malha; subamostras de $600 \mathrm{~cm}^{3}$ receberam os tratamentos. Metade das amostras de solo teve sua acidez corrigida para $\mathrm{pH}\left(\mathrm{H}_{2} \mathrm{O}\right)$ na faixa de $6,5-7,0$, utilizando-se quantidades de $\mathrm{CaCO}_{3}$ e $\mathrm{MgCO}_{3}$ (produtos p.a.), na proporção molar de 4:1, previamente definidas em ensaios de incubação. Após a adição do corretivo, as amostras foram mantidas úmidas à capacidade de campo por um período de 15 dias, quando receberam três doses de $\mathrm{Mn}$ e permaneceram incubadas por um período de 30 dias, com umidademantida próxima à capacidade de campo. O manganês foi aplicado aos solos, em solução, na forma de $\mathrm{MnCl}_{2} .4 \mathrm{H}_{2} \mathrm{O}$, nas doses de 0 , 16 e $32 \mathrm{mg} \mathrm{dm}^{-3}$. O delineamento experimental utilizado foi o inteiramente casualizado com três repetições.

Após a incubação, foram coletadas subamostras dos solos, as quais, depois de secas ao ar, foram novamente passadas em peneira de $2 \mathrm{~mm}$ de mal ha e anal isadas para $\mathrm{Mn}$ após extração com M ehlich-1

Quadro 1. Caracterização química e física das amostras de Latossolos, provenientes de Sete Lagoas (LV1), São Sebastião do Paraíso (LV2 e LV3), J oão Pinheiro (LVA1) e Viçosa (LVA2 e LVA3)

\begin{tabular}{|c|c|c|c|c|c|c|}
\hline Característica & LV1 & LV2 & LV3 & LVA1 & LVA2 & LVA3 \\
\hline $\mathrm{pH} \mathrm{H} \mathrm{H}_{2} \mathrm{O}(1: 2,5)$ & 4,6 & 4,4 & 4,7 & 4,0 & 4,1 & 4,2 \\
\hline$P\left(\mathrm{mg} \mathrm{dm}^{-3}\right)$ & 4,0 & 0,0 & 0,0 & 1,3 & 0,0 & 1,9 \\
\hline $\mathrm{K}\left(\mathrm{mg} \mathrm{dm}^{-3}\right)^{(1)}$ & 20 & 59 & 16 & 21 & 15 & 15 \\
\hline $\mathrm{Ca}^{2+}\left(\mathrm{cmol}_{\mathrm{c}} \mathrm{dm}^{-3}\right)^{(2)}$ & 1,24 & 0,55 & 0,72 & 0,08 & 0,02 & 0,46 \\
\hline $\mathrm{Mg}^{2+}\left(\mathrm{cmol}_{\mathrm{c}} \mathrm{dm}^{-3}\right)^{(2)}$ & 0,20 & 0,11 & 0,23 & 0,04 & 0,02 & 0,13 \\
\hline $\mathrm{Al}^{3+}\left(\mathrm{cmol}_{\mathrm{C}} \mathrm{dm}^{-3}\right)^{(2)}$ & 0,50 & 0,10 & 0,20 & 0,90 & 1,20 & 1,90 \\
\hline $\mathrm{H}+\mathrm{Al}\left(\mathrm{cmol}_{\mathrm{c}} \mathrm{dm}^{-3}\right)^{(3)}$ & 8,3 & 11,9 & 6,6 & 6,6 & 8,9 & 7,6 \\
\hline $\mathrm{Zn}\left(\mathrm{mg} \mathrm{dm} \mathrm{dm}^{-3}\right)^{(1)}$ & 1,21 & 0,46 & 0,37 & 0,40 & 0,18 & 1,14 \\
\hline $\mathrm{Fe}\left(\mathrm{mg} \mathrm{dm} \mathrm{dm}^{-3}\right)^{(1)}$ & 46,3 & 53,7 & 47,4 & 74,1 & 108,7 & 80,1 \\
\hline $\mathrm{Cu}\left(\mathrm{mg} \mathrm{dm}^{-3}\right)^{(1)}$ & 0,77 & 6,57 & 8,73 & 0,40 & 0,12 & 0,22 \\
\hline $\mathrm{Mn}\left(\mathrm{mg} \mathrm{dm}^{-3}\right)^{(1)}$ & 29,0 & 39,0 & 34,0 & 6,3 & 2,3 & 6,1 \\
\hline $\mathrm{CTC}_{\text {efetiva }}\left(\mathrm{cmol}_{\mathrm{c}} \mathrm{dm}^{-3}\right)$ & 2,45 & 0,91 & 1,19 & 1,07 & 1,28 & 2,53 \\
\hline $\mathrm{CTC}_{\text {total }}\left(\mathrm{cmol}_{\mathrm{c}} \mathrm{dm}^{-3}\right)$ & 10,25 & 12,71 & 7,59 & 6,77 & 8,98 & 8,23 \\
\hline V ( \%) & 14,5 & 6,4 & 13,0 & 2,5 & 0,87 & 7,7 \\
\hline m ( \%) & 20,4 & 11,0 & 16,8 & 84,1 & 93,81 & 75,1 \\
\hline C.O. $\left(\mathrm{g} \mathrm{kg}^{-1}\right)^{(4)}$ & 28,7 & 13,92 & 17,0 & 8,7 & 18,6 & 112,5 \\
\hline P-rem (mg L-1) (5) & 7,48 & 3,61 & 6,54 & 28,65 & 2,76 & 1,34 \\
\hline $\left.\mathrm{SiO}_{2}(\text { dag kg})^{-1}\right)$ & 18,3 & 4,8 & 7,4 & 12,8 & 19,8 & 10,2 \\
\hline $\mathrm{Al}_{2} \mathrm{O}_{3}\left(\text { dag kg }^{-1}\right)^{(6)}$ & 31,80 & 34,11 & 32,10 & 16,87 & 28,85 & 19,05 \\
\hline $\mathrm{Fe}_{2} \mathrm{O}_{3}\left(\mathrm{dag} \mathrm{kg}^{-1}\right)^{(6)}$ & 12,42 & 33,84 & 30,41 & 4,60 & 12,66 & 5,37 \\
\hline $\mathrm{TiO}_{2}\left(\right.$ dag kg $\left.{ }^{-1}\right)$ & 0,71 & 6,75 & 6,15 & 0,51 & 1,93 & 0,64 \\
\hline $\mathrm{MnO}\left(\text { dag kg }^{-1}\right)^{(6)}$ & 0,03 & 0,15 & 0,15 & 0,01 & 0,01 & 0,01 \\
\hline $\mathrm{P}_{2} \mathrm{O}_{5}\left(\right.$ dag kg $\left.^{-1}\right)$ & 0,21 & 0,24 & 0,22 & 0,03 & 0,06 & 0,11 \\
\hline Areia grossa $\left(\mathrm{g} \mathrm{kg}^{-1}\right)^{(7)}$ & 110 & 70 & 90 & 400 & 150 & 460 \\
\hline Areia fina $\left(\mathrm{g} \mathrm{kg}^{-1}\right)^{(7)}$ & 40 & 230 & 300 & 230 & 100 & 140 \\
\hline Silte $\left(g_{k g}^{-1}\right)^{(7)}$ & 140 & 240 & 190 & 50 & 70 & 160 \\
\hline Argila $\left(\mathrm{g} \mathrm{kg}^{-1}\right)^{(7)}$ & 710 & 460 & 420 & 320 & 680 & 240 \\
\hline
\end{tabular}

(1) Mehlich-1. ${ }^{(2)} \mathrm{KCl} 1 \mathrm{~mol} \mathrm{~L}{ }^{-1} .{ }^{(3)}$ CaOAc 0,5 mol L-1. ${ }^{(4)}$ Walkley-Black (De Filippo \& Ribeiro, 1997). ${ }^{(5)}$ Alvarez V. et al. (2000).

(6) Ataque sulfúrico (Vettori, 1969). ${ }^{(7)}$ EMBRAPA (1997). 
(De Filippo \& Ribeiro, 1997), Mehlich-3 (Mehlich, 1984), DTPA (Lindsay \& Norvell, 1978) e EDTA (Lantmann \& Meurer, 1982).

As amostras dos solos foram submetidas à extração seqüencial, baseada no método de Shuman (1985), com exceção da fração óxido de ferro amorfo, que foi obtida pelo método de Chao \& Zhou (1983). Esse fracionamento separa o $\mathrm{Mn}$ nas frações: trocável, ligada à matéria orgânica, óxido de manganês, óxido de ferro amorfo e óxido de ferro cristal ino. Os teores totais do micronutriente foram obtidos por digestão ácida deacordo comUre(1990). A fração residual foi calculada pela diferença entre os teores totais e a soma das frações anteriores.

Para avaliar a dessorção, uma mesma amostra de solo foi submetida a extrações sucessivas, utilizando-se resina catiônica. Essa técnica possi bilita dessorver praticamente toda a forma lábil desse el emento no sol o e, desse modo, quantificar o teor que poderia estar disponível às plantas em condições de cultivo. Foi utilizada uma resina trocadora de cátions, fortemente ácida (CR61CZR, I onics I nc.), em forma de lâmina, nas dimensões de 2,0 × 5,0 cm, saturada com $\mathrm{NaHCO}_{3}$.

Cada amostra de solo ( $3,000 \mathrm{~g})$ foi submetida à agitação horizontal (150 rpm) em $30 \mathrm{~mL}$ de água destilada na presença da resina em um tubo de centrífuga de $50 \mathrm{~mL}$, por um período de $16 \mathrm{~h}$, quando a resina foi retirada, lavada em água destilada com pisseta e col ocada em sol ução extratora que continha $40 \mathrm{~mL} \mathrm{HCl} 1$ mol L-1 eagitada por 2 h. As extrações sucessivas ocorreram até que a concentração do micronutriente atingisse um patamar de pequenas variações e, portanto, não houvesse dessorção adicional de $\mathrm{Mn}$ do solo. Calculou-se a quantidade total dessorvida pelo somatório dos teores obtidos nas diversas extrações. A determinação dos teores de $\mathrm{Mn}$ foi feita por espectrofotometria de absorção atômica.

\section{RESULTADOS E DISCUSSÃO}

Os teores totais de $\mathrm{Mn}$ nativo dos solos estudados encontram-se na faixa de 49 (LVA1) a $1.160 \mathrm{mg} \mathrm{kg}^{-1}$ (LV3) (Quadro 2) e estão relacionados com o material de origem, sendo encontrados maiores teores nos sol os originários de rochas máficas (LV1, LV2 eLV3) e menores nos derivados de granitos ou gnaisses (LVA1, LVA2, LVA3). Desse total, $61 \%$, em média, encontra-se em forma residual e $10 \%$ em óxidos de ferro cristalinos. Foram encontrados teores relativamente el evados em frações consideradas disponíveis, com $13 \%$ na forma trocável e $11 \%$ em formas ligadas à matéria orgânica, o que indica a baixa afinidade do $\mathrm{Mn}$ pel os sítios de adsorção mais específicos do sol o (Harter, 1991; M cBride, 1994). I sto pode ser corroborado pelo fato de que, independente- mente da reação do sol o, a fração trocável ea matéria orgânica são predominantes na retenção do el emento (Quadro 2).

Nas amostras de solo submetidas à calagem, o Mn adicionado foi transferido da forma trocável ( $9 \%$ do $\mathrm{Mn}$ total) para a matéria orgânica, os óxidos de ferro amorfo e de manganês, e fração residual. Segundo McBride (1994), os complexos orgânicos formados com o Mn são de pouca estabilidade, uma vez que o complexo formado com o ácido húmicotem caráter inteiramenteeletrostáticoe os ácidos fúlvicos apresentam limitado número de sítios de complexação específicos para o manganês. Esses fatos, aliados à baixa afinidade do elemento para formação de ligações covalentes (Harter, 1991), sugerem reposi ção para a solução do sol o mais fácil e prontamente por parte das frações orgânicas e oxídicas que a esperada no caso de micronutrientes de mais forte ligação com essas frações, como zinco e cobre, por exemplo (Nascimento, 2001).

Os maiores teores de $\mathrm{Mn}$ obtidos por todos os extratores utilizados estão relacionados com os teores totais de $\mathrm{Mn}$ nos solos, mostrando pouca dependência de características relacionadas com a capacidade-tampão, como deduzido pelos baixos coeficientes de variação obtidos para solos com ou sem calagem (Quadro 3). Os extratores mais sensíveis à mudança de $\mathrm{pH}$ foram o DTPA e o Mehlich-3. Esses resultados discordam dos dados obtidos por Abreu et al. (1994), os quais não encontraram diminuição na extração de $\mathrm{Mn}$ pelo DTPA com o aumento do pH, mas estão de acordo com os obtidos por Shuman \& Anderson (1974), que observaram esta dependência. Nos solos com calagem, os teores de $\mathrm{Mn}$ recuperados por todos os extratores foram correlacionados de forma positiva e significativa $(P<0,01)$ com os teores de matéria orgânica ( $r=0,85$ a 0,97), indicando sua partici pação na disponibilização de $\mathrm{Mn}$ em solos com $\mathrm{pH}$ mais elevado.

Vale destacar que o Mehlich-1 extraiu maiores teores de $\mathrm{Mn}$ nos solos com calagem. I sso ocorreu para todos os solos e doses, e mesmo para os solos onde não houve adição de $\mathrm{Mn}$ (Quadro 3). Esse comportamento inesperado poderia ser explicado pela distribuição do $\mathrm{Mn}$ entre as frações do solo (Quadro 2). Em sol os ácidos, 21,8 $\mathrm{mg} \mathrm{kg}^{-1}$ de $\mathrm{Mn}$, em média, estavam ligados a óxidos de fer ro cristalinos, os quais possivelmente são menos susceptíveis ao ataque ácido do Mehlich-1. Quando foi feita a calagem, os teores de $M \mathrm{n}$ nos óxidos cristalinos diminuíram para apenas 5,6 $\mathrm{mg} \mathrm{kg}^{-1}$, enquanto, nos óxidos de ferro amorfo e de manganês e na fração orgânica, aumentaram de 7,7, 15,6 e 30,0 mg kg-1 para, respectivamente, 16,8, 19,1 e35,1 $\mathrm{mg} \mathrm{kg}^{-1}$. Ou seja, em pH mais alto o $\mathrm{Mn}$ encontrou-se em formas de mais fácil dissolução pela solução ácida, mas não extraíveis pelos outros extratores, os quais nos sol os com calagem extraíram princi pal mente o Mn ligado 
Quadro 2. Concentrações de manganês nas frações: trocável (Mn-Tr), matéria orgânica (Mn-MO), óxido de manganês (Mn-OxMn), óxido de ferro amorfo (Mn-OxFeA), óxido de ferro cristalino (Mn-OxFeC), residual (Mn-R), total (Mn-T) e percentagem relativa em cada uma dessas frações em relação ao manganês total, para três doses de manganês em dois níveis de calagem

\begin{tabular}{|c|c|c|c|c|c|c|c|c|c|c|c|c|c|c|}
\hline \multirow[t]{2}{*}{ Solo } & \multirow{2}{*}{$\begin{array}{c}\text { Dose } \\
\mathrm{mg} \mathrm{dm}^{-3}\end{array}$} & \multicolumn{2}{|c|}{ Mn-Tr } & \multicolumn{2}{|c|}{ Mn-MO } & \multicolumn{2}{|c|}{ Mn-OxMn } & \multicolumn{2}{|c|}{ Mn-OxFeA } & \multicolumn{2}{|c|}{ Mn-OxFeC } & \multicolumn{2}{|c|}{ Mn-R } & \multirow{2}{*}{$\frac{\mathbf{M n}-\mathbf{T}}{\mathrm{mg} \mathrm{kg}^{-1}}$} \\
\hline & & $\mathrm{mg} \mathrm{kg}^{-1}$ & $\%$ & $\mathrm{mg} \mathrm{kg}^{-1}$ & $\%$ & $\mathrm{mg} \mathrm{kg}^{-1}$ & $\%$ & $\mathrm{mg} \mathrm{kg}^{-1}$ & $\%$ & $\mathrm{mg} \mathrm{kg}^{-1}$ & $\%$ & $\mathrm{mg} \mathrm{kg}^{-1}$ & $\%$ & \\
\hline \multicolumn{15}{|c|}{ Sem calagem } \\
\hline LV1 & $\begin{array}{r}0 \\
16 \\
32\end{array}$ & $\begin{array}{l}29,2 \\
51,3 \\
67,6\end{array}$ & $\begin{array}{l}18 \\
30 \\
35\end{array}$ & $\begin{array}{l}22,6 \\
23,0 \\
26,6\end{array}$ & $\begin{array}{l}14 \\
13 \\
14\end{array}$ & $\begin{array}{l}6,3 \\
5,3 \\
5,4\end{array}$ & $\begin{array}{l}4 \\
3 \\
3\end{array}$ & $\begin{array}{l}9,5 \\
6,9 \\
7,7\end{array}$ & $\begin{array}{l}6 \\
4 \\
4\end{array}$ & $\begin{array}{l}56,9 \\
41,2 \\
46,2\end{array}$ & $\begin{array}{l}34 \\
24 \\
24\end{array}$ & $\begin{array}{l}41,4 \\
46,2 \\
39,8\end{array}$ & $\begin{array}{l}25 \\
27 \\
21\end{array}$ & $\begin{array}{l}165,7 \\
173,9 \\
193,4\end{array}$ \\
\hline LV2 & $\begin{array}{r}0 \\
16 \\
32\end{array}$ & $\begin{array}{l}16,3 \\
46,5 \\
65,5\end{array}$ & $\begin{array}{l}2 \\
4 \\
6\end{array}$ & $\begin{array}{l}14,5 \\
19,8 \\
27,3\end{array}$ & $\begin{array}{l}1 \\
2 \\
2\end{array}$ & $\begin{array}{l}33,9 \\
29,5 \\
27,4\end{array}$ & $\begin{array}{l}3 \\
3 \\
2\end{array}$ & $\begin{array}{r}11,0 \\
9,8 \\
11,1\end{array}$ & $\begin{array}{l}1 \\
1 \\
1\end{array}$ & $\begin{array}{l}66,0 \\
58,8 \\
66,8\end{array}$ & $\begin{array}{l}6 \\
5 \\
6\end{array}$ & $\begin{array}{l}898,7 \\
924,9 \\
938,0\end{array}$ & $\begin{array}{l}86 \\
85 \\
83\end{array}$ & $\begin{array}{l}1040 \\
1089 \\
1136\end{array}$ \\
\hline LV3 & $\begin{array}{r}0 \\
16 \\
32\end{array}$ & $\begin{array}{l}30,3 \\
42,7 \\
61,1\end{array}$ & $\begin{array}{l}3 \\
4 \\
6\end{array}$ & $\begin{array}{l}80,4 \\
77,9 \\
72,6\end{array}$ & $\begin{array}{l}7 \\
7 \\
7\end{array}$ & $\begin{array}{l}53,7 \\
47,8 \\
57,1\end{array}$ & $\begin{array}{l}5 \\
4 \\
6\end{array}$ & $\begin{array}{l}19,6 \\
20,6 \\
20,4\end{array}$ & $\begin{array}{l}2 \\
2 \\
2\end{array}$ & $\begin{array}{l}1,8 \\
1,7 \\
1,7\end{array}$ & $\begin{array}{l}0 \\
0 \\
0\end{array}$ & $\begin{array}{l}974,8 \\
943,9 \\
796,9\end{array}$ & $\begin{array}{l}84 \\
83 \\
79\end{array}$ & $\begin{array}{l}1160 \\
1134 \\
1009\end{array}$ \\
\hline LVA1 & $\begin{array}{r}0 \\
16 \\
32\end{array}$ & $\begin{array}{r}6,7 \\
26,1 \\
38,7\end{array}$ & $\begin{array}{l}14 \\
44 \\
61\end{array}$ & $\begin{array}{l}1,1 \\
1,8 \\
5,3\end{array}$ & $\begin{array}{l}2 \\
3 \\
8\end{array}$ & $\begin{array}{l}0,9 \\
0,9 \\
1,0\end{array}$ & $\begin{array}{l}2 \\
2 \\
2\end{array}$ & $\begin{array}{l}0,6 \\
0,6 \\
1,1\end{array}$ & $\begin{array}{l}1 \\
1 \\
2\end{array}$ & $\begin{array}{l}3,9 \\
3,7 \\
9,5\end{array}$ & $\begin{array}{r}8 \\
6 \\
15\end{array}$ & $\begin{array}{r}35,7 \\
25,5 \\
8,2\end{array}$ & $\begin{array}{l}73 \\
43 \\
13\end{array}$ & $\begin{array}{l}48,9 \\
58,6 \\
63,9\end{array}$ \\
\hline LVA2 & $\begin{array}{r}0 \\
16 \\
32\end{array}$ & $\begin{array}{l}14,3 \\
25,5 \\
41,7\end{array}$ & $\begin{array}{l}19 \\
31 \\
42\end{array}$ & $\begin{array}{l}3,6 \\
4,9 \\
6,4\end{array}$ & $\begin{array}{l}5 \\
6 \\
6\end{array}$ & $\begin{array}{l}0,1 \\
0,1 \\
0,1\end{array}$ & $\begin{array}{l}0 \\
0 \\
0\end{array}$ & $\begin{array}{l}0,5 \\
0,4 \\
0,4\end{array}$ & $\begin{array}{l}1 \\
1 \\
0\end{array}$ & $\begin{array}{l}2,7 \\
2,7 \\
3,3\end{array}$ & $\begin{array}{l}3 \\
3 \\
3\end{array}$ & $\begin{array}{l}54,6 \\
47,9 \\
47,8\end{array}$ & $\begin{array}{l}72 \\
59 \\
48\end{array}$ & $\begin{array}{l}75,9 \\
81,6 \\
99,8\end{array}$ \\
\hline LVA3 & $\begin{array}{r}0 \\
16 \\
32\end{array}$ & $\begin{array}{r}20,4 \\
55,9 \\
110,3\end{array}$ & $\begin{array}{l}20 \\
36 \\
49\end{array}$ & $\begin{array}{l}35,4 \\
51,8 \\
65,8\end{array}$ & $\begin{array}{l}35 \\
33 \\
29\end{array}$ & $\begin{array}{l}2,8 \\
3,8 \\
4,3\end{array}$ & $\begin{array}{l}3 \\
2 \\
2\end{array}$ & $\begin{array}{l}3,4 \\
6,8 \\
7,5\end{array}$ & $\begin{array}{l}3 \\
4 \\
3\end{array}$ & $\begin{array}{l}9,9 \\
7,9 \\
7,4\end{array}$ & $\begin{array}{r}10 \\
5 \\
3\end{array}$ & $\begin{array}{l}27,9 \\
30,8 \\
28,9\end{array}$ & $\begin{array}{l}28 \\
20 \\
13\end{array}$ & $\begin{array}{r}99,7 \\
157,1 \\
224,2\end{array}$ \\
\hline Média & & 41,7 & 23 & 30,0 & 11 & 15,6 & 2,5 & 7,7 & 2 & 21,8 & 9 & 328,4 & 52 & 445 \\
\hline C.V. & & 3,7 & & 6,3 & & 12,9 & & 12,2 & & 10,6 & & 10,1 & & 7,4 \\
\hline \multicolumn{15}{|c|}{ Com calagem } \\
\hline LV1 & $\begin{array}{r}0 \\
16 \\
32\end{array}$ & $\begin{array}{l}15,1 \\
22,5 \\
28,6\end{array}$ & $\begin{array}{r}9 \\
13 \\
15\end{array}$ & $\begin{array}{l}35,5 \\
60,9 \\
76,0\end{array}$ & $\begin{array}{l}21 \\
35 \\
39\end{array}$ & $\begin{array}{r}5,2 \\
11,7 \\
14,3\end{array}$ & $\begin{array}{l}3 \\
7 \\
7\end{array}$ & $\begin{array}{l}27,1 \\
25,4 \\
27,3\end{array}$ & $\begin{array}{l}16 \\
15 \\
14\end{array}$ & $\begin{array}{l}10,6 \\
12,6 \\
15,3\end{array}$ & $\begin{array}{l}6 \\
7 \\
8\end{array}$ & $\begin{array}{l}72,2 \\
40,7 \\
31,9\end{array}$ & $\begin{array}{l}44 \\
23 \\
16\end{array}$ & $\begin{array}{l}165,7 \\
173,9 \\
193,4\end{array}$ \\
\hline LV2 & $\begin{array}{r}0 \\
16 \\
32\end{array}$ & $\begin{array}{l}6,7 \\
7,6 \\
9,3\end{array}$ & $\begin{array}{l}1 \\
1 \\
1\end{array}$ & $\begin{array}{l}24,8 \\
31,1 \\
37,9\end{array}$ & $\begin{array}{l}2 \\
3 \\
3\end{array}$ & $\begin{array}{l}59,1 \\
63,4 \\
84,7\end{array}$ & $\begin{array}{l}6 \\
6 \\
7\end{array}$ & $\begin{array}{l}20,7 \\
21,7 \\
21,9\end{array}$ & $\begin{array}{l}2 \\
2 \\
2\end{array}$ & $\begin{array}{l}0,4 \\
0,3 \\
0,4\end{array}$ & $\begin{array}{l}0 \\
0 \\
0\end{array}$ & $\begin{array}{l}928,3 \\
964,9 \\
981,8\end{array}$ & $\begin{array}{l}89 \\
89 \\
86\end{array}$ & $\begin{array}{l}1040 \\
1089 \\
1136\end{array}$ \\
\hline LV3 & $\begin{array}{r}0 \\
16 \\
32\end{array}$ & $\begin{array}{l}11,6 \\
18,8 \\
22,9\end{array}$ & $\begin{array}{l}1 \\
2 \\
2\end{array}$ & $\begin{array}{l}73,1 \\
83,6 \\
86,7\end{array}$ & $\begin{array}{l}6 \\
7 \\
9\end{array}$ & $\begin{array}{l}25,3 \\
23,6 \\
23,7\end{array}$ & $\begin{array}{l}2 \\
2 \\
2\end{array}$ & $\begin{array}{l}27,8 \\
27,1 \\
27,9\end{array}$ & $\begin{array}{l}2 \\
2 \\
3\end{array}$ & $\begin{array}{l}3,1 \\
3,1 \\
1,5\end{array}$ & $\begin{array}{l}0 \\
0 \\
0\end{array}$ & $\begin{array}{l}1019,1 \\
977,8 \\
846,3\end{array}$ & $\begin{array}{l}88 \\
86 \\
84\end{array}$ & $\begin{array}{l}1160 \\
1134 \\
1009\end{array}$ \\
\hline LVA1 & $\begin{array}{r}0 \\
16 \\
32\end{array}$ & $\begin{array}{l}0,3 \\
4,5 \\
9,2\end{array}$ & $\begin{array}{r}1 \\
8 \\
14\end{array}$ & $\begin{array}{l}0,3 \\
1,2 \\
8,5\end{array}$ & $\begin{array}{r}1 \\
2 \\
13\end{array}$ & $\begin{array}{l}1,6 \\
3,6 \\
4,5\end{array}$ & $\begin{array}{l}3 \\
6 \\
7\end{array}$ & $\begin{array}{l}3,0 \\
4,5 \\
6,2\end{array}$ & $\begin{array}{r}6 \\
8 \\
10\end{array}$ & $\begin{array}{l}7,5 \\
6,1 \\
6,5\end{array}$ & $\begin{array}{l}15 \\
10 \\
10\end{array}$ & $\begin{array}{l}36,2 \\
38,7 \\
29,0\end{array}$ & $\begin{array}{l}74 \\
66 \\
45\end{array}$ & $\begin{array}{l}48,9 \\
58,6 \\
63,9\end{array}$ \\
\hline LVA2 & $\begin{array}{r}0 \\
16 \\
32\end{array}$ & $\begin{array}{r}0,5 \\
15,5 \\
29,7\end{array}$ & $\begin{array}{r}1 \\
19 \\
30\end{array}$ & $\begin{array}{r}2,0 \\
7,4 \\
13,1\end{array}$ & $\begin{array}{r}3 \\
9 \\
13\end{array}$ & $\begin{array}{l}0,7 \\
1,7 \\
2\end{array}$ & $\begin{array}{l}1 \\
2 \\
2\end{array}$ & $\begin{array}{l}2,4 \\
2,6 \\
4,3\end{array}$ & $\begin{array}{l}3 \\
3 \\
4\end{array}$ & $\begin{array}{l}1,4 \\
1,7 \\
2,0\end{array}$ & $\begin{array}{l}2 \\
2 \\
2\end{array}$ & $\begin{array}{l}68,9 \\
52,7 \\
48,7\end{array}$ & $\begin{array}{l}91 \\
65 \\
49\end{array}$ & $\begin{array}{l}75,9 \\
81,6 \\
99,8\end{array}$ \\
\hline LVA3 & $\begin{array}{r}0 \\
16 \\
32\end{array}$ & $\begin{array}{r}5,5 \\
23,8 \\
41,1\end{array}$ & $\begin{array}{r}6 \\
15 \\
18\end{array}$ & $\begin{array}{l}17,1 \\
31,4 \\
41,4\end{array}$ & $\begin{array}{l}17 \\
20 \\
18\end{array}$ & $\begin{array}{l}4,2 \\
6,4 \\
8,2\end{array}$ & $\begin{array}{l}4 \\
4 \\
4\end{array}$ & $\begin{array}{l}12,4 \\
18,6 \\
21,4\end{array}$ & $\begin{array}{l}12 \\
12 \\
10\end{array}$ & $\begin{array}{l}9,3 \\
9,5 \\
9,2\end{array}$ & $\begin{array}{l}9 \\
6 \\
4\end{array}$ & $\begin{array}{r}51,2 \\
67,4 \\
102,9\end{array}$ & $\begin{array}{l}51 \\
43 \\
46\end{array}$ & $\begin{array}{r}99,7 \\
157,1 \\
224,2\end{array}$ \\
\hline Média & & 15,2 & 9 & 35,1 & 12 & 19,1 & 4 & 16,8 & 7 & 5,6 & 5 & 353,3 & 63 & 445 \\
\hline C.V. (\%) & & 7,3 & & 6,7 & & 8,2 & & 8,4 & & 10,1 & & 5,0 & & 7,4 \\
\hline
\end{tabular}

às formas: trocável e matéria orgânica. Isto indica uma inadequabilidade do uso do Mehlich-1 para avaliação da disponibilidade de $\mathrm{Mn}$ em solos submetidos à calagem.

Nos solos não submetidos à calagem, o $\mathrm{Mn}$ encontrou-se, predominantemente, em forma trocável (Quadro 2). N essa condição, o Mehlich-3 foi o extrator que apresentou a mel hor correlação com essa fração (Quadro 4). No entanto, para a fração matéria orgânica, a correlação com esse extrator foi menor que as obtidas pelo Mehlich-1 e EDTA. Quando feita a cal agem, condição em que a matéria orgânica e os óxidos de ferro amorfo e de manganês passam, juntamente com a fração trocável, a reter o 
Quadro 3. Manganês extraído por Mehlich-1, Mehlich-3, DTPA e EDTA de amostras de seis Latossolos de Minas Gerais em três doses de manganês e dois níveis de calagem e as respectivas declividades Mn recuperado/Mn adicionado $\left(\mathrm{mg} \mathrm{dm}^{-3} / \mathrm{mg} \mathrm{dm}^{-3}\right)$

\begin{tabular}{|c|c|c|c|c|c|c|c|c|c|}
\hline \multirow[t]{2}{*}{ Solo } & \multirow{2}{*}{$\begin{array}{c}\text { Dose } \\
\mathrm{mg} \mathrm{dm}^{-3}\end{array}$} & \multicolumn{2}{|c|}{ Mehlich-1 } & \multicolumn{2}{|c|}{ Mehlich-3 } & \multicolumn{2}{|c|}{ DTPA } & \multicolumn{2}{|c|}{ EDTA } \\
\hline & & $\mathrm{mg} \mathrm{dm}^{-3}$ & Decliv. & $\mathrm{mg} \mathrm{dm} \mathrm{m}^{-3}$ & Decliv. & $\mathrm{mg} \mathrm{dm}^{-3}$ & Decliv. & $\mathrm{mg} \mathrm{dm}^{-3}$ & Decliv. \\
\hline LV1 & $\begin{array}{r}0 \\
16 \\
32\end{array}$ & $\begin{array}{l}39,83 \\
55,41 \\
63,28\end{array}$ & 0,73 & $\begin{array}{l}\quad \text { Sem } \\
28,42 \\
47,46 \\
65,66\end{array}$ & lagem & $\begin{array}{l}21,01 \\
42,21 \\
54,41\end{array}$ & 1,04 & $\begin{array}{l}33,67 \\
59,42 \\
72,65\end{array}$ & 1,22 \\
\hline LV2 & $\begin{array}{r}0 \\
16 \\
32\end{array}$ & $\begin{array}{l}35,98 \\
48,81 \\
66,96\end{array}$ & 0,97 & $\begin{array}{l}26,82 \\
50,88 \\
74,29\end{array}$ & 1,48 & $\begin{array}{l}15,19 \\
38,65 \\
60,41\end{array}$ & 1,41 & $\begin{array}{r}61,12 \\
77,89 \\
101,82\end{array}$ & 1,27 \\
\hline LV3 & $\begin{array}{r}0 \\
16 \\
32\end{array}$ & $\begin{array}{l}53,33 \\
74,57 \\
92,88\end{array}$ & 1,23 & $\begin{array}{l}44,08 \\
65,17 \\
72,12\end{array}$ & 0,88 & $\begin{array}{l}21,71 \\
33,52 \\
48,54\end{array}$ & 0,84 & $\begin{array}{l}108,74 \\
135,36 \\
147,86\end{array}$ & 1,22 \\
\hline LVA1 & $\begin{array}{r}0 \\
16 \\
32\end{array}$ & $\begin{array}{r}7,83 \\
20,28 \\
35,33\end{array}$ & 0,86 & $\begin{array}{r}5,63 \\
23,03 \\
44,84\end{array}$ & 1,22 & $\begin{array}{r}5,86 \\
22,01 \\
41,05\end{array}$ & 1,10 & $\begin{array}{r}3,63 \\
32,35 \\
55,38\end{array}$ & 1,62 \\
\hline LVA2 & $\begin{array}{r}0 \\
16 \\
32\end{array}$ & $\begin{array}{r}3,43 \\
13,16 \\
23,56\end{array}$ & 0,63 & $\begin{array}{r}1,38 \\
14,38 \\
30,47\end{array}$ & 0,91 & $\begin{array}{r}1,13 \\
10,70 \\
21,47\end{array}$ & 0,73 & $\begin{array}{r}1,38 \\
20,50 \\
36,90\end{array}$ & 1,11 \\
\hline LVA3 & $\begin{array}{r}0 \\
16 \\
32\end{array}$ & $\begin{array}{l}17,84 \\
31,02 \\
45,56\end{array}$ & 0,87 & $\begin{array}{r}7,92 \\
32,70 \\
53,02\end{array}$ & 1,41 & $\begin{array}{r}1,38 \\
5,72 \\
13,36\end{array}$ & 0,37 & $\begin{array}{r}5,60 \\
37,10 \\
65,75\end{array}$ & 1,89 \\
\hline Média & & 40,50 & 0,88 & 38,24 & 1,18 & 25,46 & 0,91 & 58,73 & 1,39 \\
\hline C.V. (\%) & & 5,06 & & 4,81 & & 7,75 & & 5,54 & \\
\hline LV1 & $\begin{array}{r}0 \\
16 \\
32\end{array}$ & $\begin{array}{l}50,10 \\
68,05 \\
82,17\end{array}$ & 1,00 & $\begin{array}{r}\text { Com } \\
14,67 \\
25,89 \\
42,39\end{array}$ & 0,87 & $\begin{array}{r}8,17 \\
13,86 \\
18,68\end{array}$ & 0,33 & $\begin{array}{l}17,40 \\
41,38 \\
56,23\end{array}$ & 1,21 \\
\hline LV2 & $\begin{array}{r}0 \\
16 \\
32\end{array}$ & $\begin{array}{r}81,04 \\
102,23 \\
103,48\end{array}$ & 0,70 & $\begin{array}{l}28,25 \\
41,35 \\
53,06\end{array}$ & 0,77 & $\begin{array}{l}4,91 \\
5,13 \\
7,16\end{array}$ & 0,07 & $\begin{array}{l}42,05 \\
65,50 \\
86,42\end{array}$ & 1,39 \\
\hline LV3 & $\begin{array}{r}0 \\
16 \\
32\end{array}$ & $\begin{array}{r}87,06 \\
97,91 \\
105,43\end{array}$ & 0,57 & $\begin{array}{l}36,26 \\
45,16 \\
59,74\end{array}$ & 0,73 & $\begin{array}{r}8,21 \\
12,49 \\
15,74\end{array}$ & 0,23 & $\begin{array}{l}109,38 \\
132,09 \\
150,28\end{array}$ & 1,28 \\
\hline LVA1 & $\begin{array}{r}0 \\
16 \\
32\end{array}$ & $\begin{array}{l}10,33 \\
29,67 \\
50,08\end{array}$ & 1,24 & $\begin{array}{r}5,16 \\
17,01 \\
33,31\end{array}$ & 0,88 & $\begin{array}{r}1,63 \\
7,10 \\
10,01\end{array}$ & 0,26 & $\begin{array}{r}2,23 \\
31,30 \\
52,17\end{array}$ & 1,56 \\
\hline LVA2 & $\begin{array}{r}0 \\
16 \\
32\end{array}$ & $\begin{array}{r}4,80 \\
19,46 \\
34,92\end{array}$ & 0,94 & $\begin{array}{r}0,12 \\
16,13 \\
28,41\end{array}$ & 0,88 & $\begin{array}{r}0,61 \\
8,48 \\
20,10\end{array}$ & 0,61 & $\begin{array}{r}1,52 \\
17,78 \\
39,46\end{array}$ & 1,18 \\
\hline LVA3 & $\begin{array}{r}0 \\
16 \\
32\end{array}$ & $\begin{array}{l}29,81 \\
60,30 \\
92,38\end{array}$ & 1,95 & $\begin{array}{r}6,67 \\
30,05 \\
55,14\end{array}$ & 1,51 & $\begin{array}{r}5,90 \\
29,64 \\
53,69\end{array}$ & 1,49 & $\begin{array}{r}3,98 \\
38,01 \\
71,88\end{array}$ & 2,12 \\
\hline Média & & 61,62 & 1,06 & 29,93 & 0,94 & 12,86 & 0,49 & 53,28 & 1,46 \\
\hline C.V. (\%) & & 3,19 & & 5,72 & & 11,23 & & 6,39 & \\
\hline
\end{tabular}

$\mathrm{Mn}$, as mel hores correlações com essas frações foram obtidas pelo Mehlich-1. O DTPA, com exceção da fração trocável nos sol os com calagem, foi o extrator que apresentou as menores correlações com as frações de manganês.

Em relaçãoà dessorção (Quadro 5), para os solos não submetidos à calagem, os maiores teores recuperados pela resina, nas doses de 0 e $16 \mathrm{mg} \mathrm{dm}^{-3}$, foram obtidos para o LV1 e, na doses de $32 \mathrm{mg} \mathrm{dm}^{-3}$, para oLVA3. Com a cal agem, a resina dessorveu mais $\mathrm{Mn}$ do LV3 (doses 0 e $16 \mathrm{mg} \mathrm{dm}^{-3}$ ) e do LVA3 (dose $32 \mathrm{mg} \mathrm{dm}^{-3}$ ). Comparando esses dados com os do fracionamento (Quadro 2), percebe-se que o $\mathrm{Mn}$ recuperado pela resina foi quase inteiramente composto pel o Mn trocável, uma vez que os sol os que mostraram maior dessorção foram os mesmos que 
Quadro 4. Coeficientes de correlação linear simples entre os teores de manganês por Mehlich-1, Mehlich-3, DTPA e EDTA e as frações desse micronutriente ( $\mathrm{Tr}$ - trocável, MO - matéria orgânica, OxMn - óxido de manganês, OxFeA - óxido de ferro amorfo, OxFeC - óxido de ferro cristalino, $\mathbf{R}$ - residual e T total) de amostras de seis solos, para dois níveis de calagem

\begin{tabular}{|c|c|c|c|c|c|c|c|c|}
\hline Extrator & Calagem & Mn-Tr & Mn-MO & $M n-O x M n$ & Mn-OxFeA & Mn-OxFeC & $M n-R$ & Mn-T \\
\hline Mehich -1 & $\begin{array}{l}S / C \\
C / C\end{array}$ & $\begin{array}{l}0,58^{* *} \\
0,41^{* *}\end{array}$ & $\begin{array}{l}0,68^{* *} \\
0,78^{* *}\end{array}$ & $\begin{array}{l}0,73^{* *} \\
0,68^{* *}\end{array}$ & $\begin{array}{l}0,84^{* *} \\
0,85^{* *}\end{array}$ & $\begin{array}{c}0,28^{*} \\
-0,12 \text { NS }\end{array}$ & $\begin{array}{l}0,62^{* *} \\
0,73^{* *}\end{array}$ & $\begin{array}{l}0,68^{* *} \\
0,78^{* *}\end{array}$ \\
\hline Mehich-3 & $\begin{array}{l}S / C \\
C / C\end{array}$ & $\begin{array}{l}0,73 * * \\
0,59 * *\end{array}$ & $\begin{array}{l}0,56 * * \\
0,69^{* *}\end{array}$ & $\begin{array}{l}0,57^{* *} \\
0,52^{* *}\end{array}$ & $\begin{array}{l}0,68^{* *} \\
0,65^{* *}\end{array}$ & $\begin{array}{c}0,30 * \\
-0,14 \text { NS }\end{array}$ & $\begin{array}{l}0,53^{* *} \\
0,58^{* *}\end{array}$ & $\begin{array}{l}0,60^{* *} \\
0,63^{* *}\end{array}$ \\
\hline DTPA & $\begin{array}{l}S / C \\
C / C\end{array}$ & $\begin{array}{l}0,46 * * \\
0,87^{* *}\end{array}$ & $\begin{array}{l}0,15 \mathrm{Ns} \\
0,27 \mathrm{Ns}\end{array}$ & $\begin{array}{r}0,39 * * \\
-0,20 \text { Ns }\end{array}$ & $\begin{array}{l}0,43^{* *} \\
0,26 \mathrm{NS}\end{array}$ & $\begin{array}{l}0,45^{* *} \\
0,35^{* *}\end{array}$ & $\begin{array}{r}0,41^{* *} \\
-0,23 \text { NS }\end{array}$ & $\begin{array}{r}0,45^{* *} \\
-0,17 \text { Ns }\end{array}$ \\
\hline EDTA & $\begin{array}{l}S / C \\
C / C\end{array}$ & $\begin{array}{l}0,48 * * \\
0,36 * *\end{array}$ & $\begin{array}{l}0,72^{* *} \\
0,81^{* *}\end{array}$ & $\begin{array}{l}0,86^{* *} \\
0,43^{* *}\end{array}$ & $\begin{array}{l}0,89 * * \\
0,65 * *\end{array}$ & $\begin{array}{c}0,13 \text { Ns } \\
-0,30^{* *}\end{array}$ & $\begin{array}{l}0,77 * * \\
0,76 * *\end{array}$ & $\begin{array}{l}0,81 * * \\
0,80^{* *}\end{array}$ \\
\hline
\end{tabular}

*i**e ${ }^{\mathrm{NS}}$ : Significativos a 5 e $1 \%$, respectivamente, e não-significativo.

Quadro 5. Manganês recuperado por extrações sucessivas com resina catiônica para três doses do elemento aplicadas a amostras de Latossolos, para dois níveis calagem

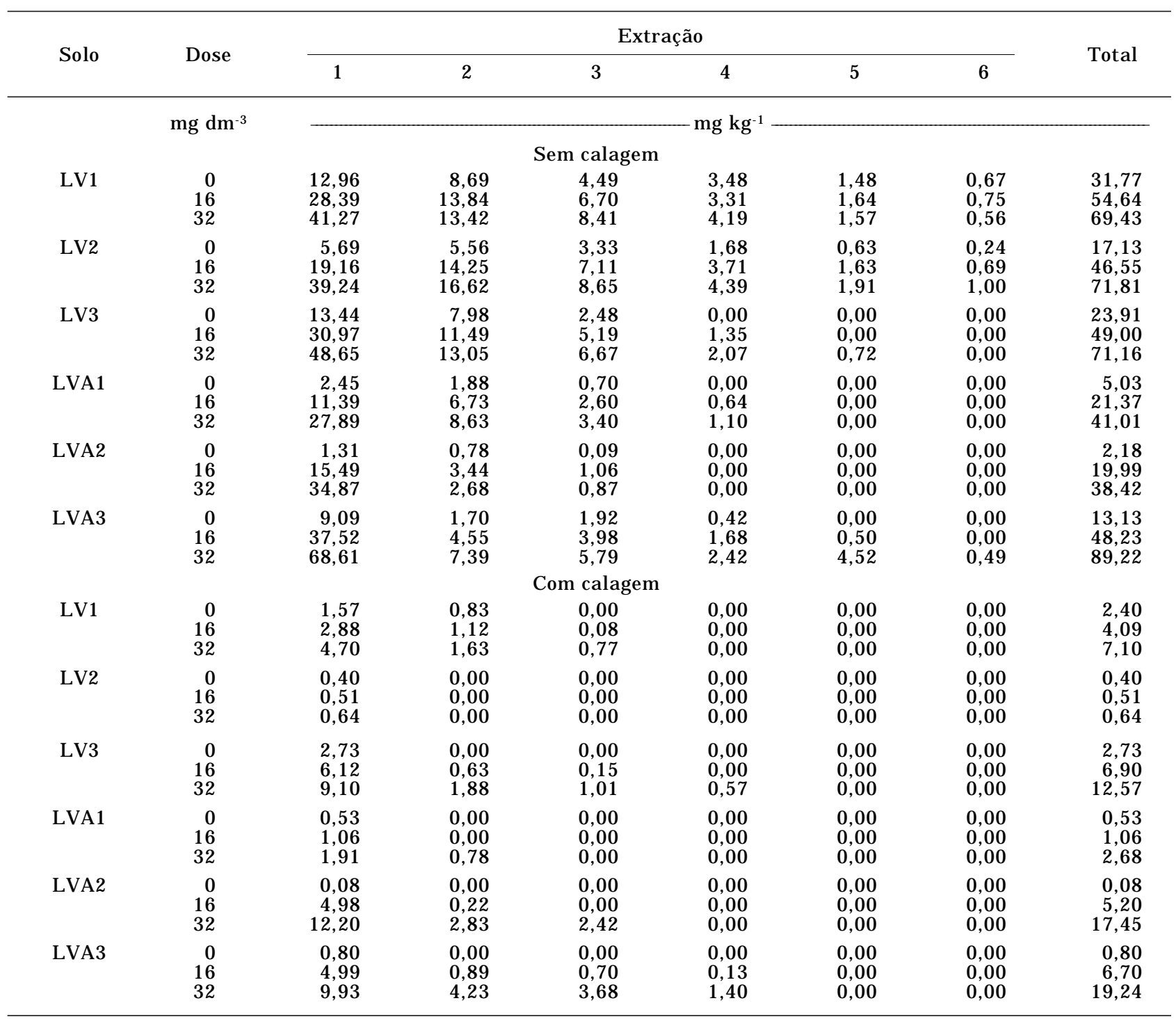


retiveram $M n$ em maior proporção na fração trocável. Com o aumento do $\mathrm{pH}$, percebeu-se acentuada diminui ção dos teores de $\mathrm{Mn}$ dessorvidos para todos os solos e doses, indicando a passagem do elemento para formas de menor solubilidade, preci pitadas ou ligadas a óxidos, como indicado pelo fracionamento.

N os sol os sem cal agem, a mel hor correlação entre os teores dessorvidos e os obtidos pelos extratores foi para o M ehlich-3, enquanto, após a cal agem, o DTPA foi o extrator que estimou mais satisfatoriamente o Mn dessorvido pela resina (Quadro 6). I sso deveuse ao fato de a capacidade de cada um desses extratores, em cada situação, ser mais bem correlacionada, com o Mn trocável (Quadro 4) e, portanto, representar o teor lábil do elemento. O Mehlich-1 não se correlacionou com o Mn dessorvido nos solos que receberam calagem, novamente indicando não ser esse extrator capaz de discriminar os teores de Mn lábil em sol os com a acidez corrigida.

\section{CONCLUSÕES}

1. Com a aplicação do manganês nos solos, o elemento foi retido, principalmente, na fração trocável e matéria orgânica. De modo geral, a calagem provocou redução nos teores trocáveis eaumento nas frações óxidos de ferro amorfo e de manganês.

2. A ordem de dessorção apresentada pelo manganês, na ausência de calagem, foi $\operatorname{Tr} \gg>$ MO > OxFeA. A calagem provocou acentuado decréscimo nos teores de $\mathrm{Mn}$ dessorvidos.

Quadro 6. Correlações lineares simples entre os teores totais de Mn dessorvidos, as frações e teores extraídos por Mehlich-1, Mehlich-3, DTPA e EDTA, em seis amostras de Latossolos submetidos a três doses de manganês, sem e com calagem

\begin{tabular}{lcc} 
F ração e extrator & Sem calagem & Com calagem \\
\hline Trocável & $0,95^{* *}$ & $0,89^{* *}$ \\
M.O. & $0,49^{* *}$ & $0,21^{*}$ \\
ÓxidoMn & $0,23^{\mathrm{NS}}$ & $-0,27^{*}$ \\
ÓxidoFeA & $0,41^{* *}$ & $0,14^{\mathrm{NS}}$ \\
ÓxidoFeC & $0,22^{\mathrm{NS}}$ & $0,10^{\mathrm{NS}}$ \\
Residual & $0,18^{\mathrm{NS}}$ & $-0,11^{\mathrm{NS}}$ \\
Total & $0,27^{\mathrm{NS}}$ & $-0,07^{\mathrm{NS}}$ \\
& & \\
Mehlich-1 & $0,75^{* *}$ & $0,25^{\mathrm{NS}}$ \\
Mehlich-3 & $0,87^{* *}$ & $0,51^{* *}$ \\
DTPA & $0,67^{* *}$ & $0,82^{* *}$ \\
EDTA & $0,63^{* *}$ & $0,36^{* *}$ \\
\hline
\end{tabular}

*i**e $e^{\text {NS}}$ : Significativos a 5 e $1 \%$, respectivamente enão-significativo.
3. O Mehlich-1 mostrou-se inadequado para a determinação dos teores disponíveis de $\mathrm{Mn}$ em sol os corrigidos, condição na qual o DTPA foi o extrator mais indicado.

\section{LITE RATURA CITADA}

ABREU, C.A.; NOVAIS, R.F.; RAIJ , B. van \& RIBEIRO, A.C. Influência da reação do solo na extração de manganês por diferentes extratores químicos. R. Bras. Ci. Solo 18:91-99, 1994.

ALLOWAY, B.J. The origins of heavy metals in soils. In: ALLOWAY, B.J ., ed. Heavy metals in soils. New York, J ohn Wiley \& Sons, 1990. p.29-39.

ALVAREZ V, V.H.; NOVAIS, R.F.; DIAS, L.E. \& OLIVEIRA, J.A. Determinação e uso do fósforo remanescente. B. I nf. SBCS, 25:21-32, 2000

BIBAK, A. Competitive sorption of copper, nickel, and zinc by an Oxisol. Comm. Soil Sci. Plant Anal., 28:927-937, 1997.

CHAO, T.T. \& ZHOU, L. Extraction techniques for selective dissolution of amorphous iron oxides from soils and sediments. Soil Sci. Soc. Am. J ., 47:224-232, 1983.

De FILIPPO, B.V. \& RIBEIRO, A.C. Análise química do solo (metodologia - $2^{a}$ edição). Viçosa, Universidade Federal de Viçosa, 1997. $26 \mathrm{p}$.

EMPRESA BRASILEIRA DE PESQUISA AGROPECUÁRIA EMBRAPA. Manual de métodos de análise de solo. 2. ed. Rio deJ aneiro, Centro Nacional de Pesquisa deSolos, 1997. $212 \mathrm{p}$.

HARTER, R.D. Micronutrient adsorption-desorption reactions in soils. In: MORTVERDT, J .J .; COX, F.R.; SHUMAN, L.M. \& WELCH, R.M., eds. Micronutrients in the agriculture, Madison, Soil Science Society of America, 1991. p.59-88.

HOGG, D.S.; MCLAREN, R.G. \& SWIFT, R.S. Desorption of copper from some New Zealand Soils. Soil Sci. Soc. Am. J ., 57:361366, 1993.

LANTMANN, A.F. \& MEURER, E.J. Estudo da eficiência de extratores para avaliação do zinco disponível do solo para o milho. R. Bras. Ci. Solo, 6:131-135, 1982.

LINDSAY, W.L. \& NORVELL, W.A. Development of a DTPA soil test for zinc, iron, manganese and copper. Soil Sc. Soc. Am. J ., 42:421-428, 1978.

McBRIDE, M.B. Environmental chemistry of soils. New York, Oxford University Press, 1994. 406p.

MEHLICH, A. Mehlich-3 soil test extractant: a modification of Mehlich-2 extractant. Comm. Soil Sci. Plant Anal., 15:14091416, 1984.

MESQUITA, M.E. Copper and zinc competitive adsorption in schistic and granitic acid soils. Agrochimica, 17:235-244, 1998.

MSAKY, J.J \& \& CALVET, R. Adsorption behavior of copper and zinc in soils: influence of $\mathrm{pH}$ on adsorption characteristics. Soil Sci., 150:513-522, 1990. 
NASCIMENTO, C.W.A. Dessorção, extração e fracionamento de zinco, cobre e manganês em solos. Viçosa, Universidade Federal de Viçosa, 2001. 60 p. (Tese de Doutorado)

SHUMAN, L.M. \& ANDERSON, O.E. Evaluation of six extractants for their ability to predict manganese concentrations in wheat and soybeans. Soil Sc. Soc. Am. Proc., 38:788-790, 1974.

SHUMAN, L.M. The effect of soil properties on zinc adsorption by soils. Soil Sci. Soc. Am. Proc., 39:454-458, 1975.

SHUMAN, L.M. Fractionation method for soil microelements. Soil Sci., 140:11-22, 1985.

SIMS, J.T. \& J OHNSON, G.V. Micronutrients soil tests. In: MORTVERDT, J J .; COX, F.R.; SHUMAN, L.M. \& WELCH, R.M., eds. Micronutrients in agriculture, Madison, Soil Science Society of America, 1991. p.427-472.
SWIFT, R.S. \& MCLAREN, R.G. Micronutrient sorption by soils and soil colloids. In: BOLT, G.H., ed. Interactions at the soil colloid-soil solution interface. Dordrecht, Kluwer Academic, 1991. p.257-292.

URE, A. M. Methods of analysis for heavy metals in soils. In: ALLOWAY, B.J ., ed. Heavy metals in soils. New York, J ohn Wiley \& Sons, 1990. p.40-80.

VETTORI, L. Métodos de análise de solo. Rio de J aneiro, Ministério da Agricultura, Equipe dePedologia eFertilidade do Solo, 1969. 24p. (Boletim técnico, 7)

YUAN, G. \& LAVKULICH, L.M. Sorption behavior of copper, zinc, and cadmium in response to simulated changes in soils properties. Comm. Soil Sci. Plant. Anal., 28:571-587, 1997.

ZHU, B. \& ALVA, A.K. The chemical forms of $\mathrm{Zn}$ and $\mathrm{Cu}$ extractable by Mehlich-1, Mehlich-3, and ammonium bicarbonate-DTPA extractions. Soil Sci., 156:251-258, 1993. 
C.W.A. NASCIMENTO et al.

R. Bras. Ci. Solo, 26:589-597, 2002 